\title{
Da fortezza a residenza castellana: osservazioni stratigrafiche per la comprensione del processo trasformativo della Rocca di Novellara (RE, Italia)
}

From a fortress to a residential castle: a stratigraphic reading of the transformations in the Rocca of Novellara (RE, Italy)

\section{Angela Squassina}

Università IUAV di Venezia, Venice, Italy, squassin@iuav.it

\begin{abstract}
The paper reports the results of a stratigraphic reading on the northern façade of the Rocca di Novellara (Reggio Emilia, Italy), a castle which is now the town hall, right in the city centre. Though as a pole of the contemporary public life in Novellara, housing at present both a museum and a nineteenth century theatre, the Rocca recalls its military past through its name and by means of the still standing remains of the walls and corner towers. Besides a well-documented historical development, the stratigraphic investigation of the northern façade -the only part that still hasn't been restored-allowed a direct observation of the material traces revealing the slow transformation of the Rocca from a fortification to a residential castle. This study gave the chance of understanding the different constructive phases of the castle, making a chronological sequence out of them but it was also meant to reflect about the changes of its character, as the building has been acquiring a complex identity through time, due both to high qualified architectural episodes and to as much meaningful though tiny changes. Thus, the permanence of the stratified marks can be regarded as one of the main goals of a preservation project.
\end{abstract}

Keywords: Fortress, preservation, stratigraphy, traces.

\section{Introduzione. Le origini: dalla curtis al Castellunculum (X-XIV secolo)}

"Fu nell'unX secolo che Novellara dall'essere di Corte o di villaggio avente la sua Pieve [...] passò a quello di Borgo e di Castello. Fu essa compresa fra i domini di Gherardaccio Malapresa Signore Longobardo [...] aumentò questo il primo borgo di Novellara, gli scavò d'intorno le fosse, lo circondò di terrapieni ed una alta e forte torre vi innalzò a sua difesa nel luogo appunto, ove è oggi il piazzale della Rocca" (Davolio, 1825 , p. 15, fig. 1). La descrizione del primo nucleo di Novellara è significativa per comprendere l'evoluzione dell'insediamento, a partire da un assetto a corte che fu ampliato a formare una sorta di castello-recinto, (forse un castelliere, Perogalli, 1972, pp. 16-17), espressione del fenomeno dell'incastellamento, sviluppatosi a partire dal X secolo dopo l'invasione degli Ungari, come ricorda il canonico Vincenzo Davolio (1825, p. 15). La definizione del primitivo $\mathrm{Ca}$ stellunculum compare in un atto di vendita del 1142, stilato dai Malapresa (Barilli, 1999, p. 11); mentre la sua raffigurazione come un insieme di edifici circondato da una palizzata figura nella più antica rappresentazione cartografica di Novellara risalente al 1449 (Barilli, 1999, p. 11). "Consisteva questa [fortificazione] nell' alta torre 
già mentovata di sopra, circondata da ristretto quadrato di bassi fabbricati, che servivano per alloggiamento de soldati destinati a difenderlo. Era questa la forma ordinaria de fortilizii di quei tempi, [...] fino all'invenzione della polvere e dell'Artiglieria [...]" (Davolio, 1825, p. 31).

Un'ulteriore specificazione cronologica e descrittiva la colloca "[...] agli inizi del XII secolo [quando] Gherardo Malaspina [Malapresa ndr] ${ }^{1}$ fece alzare una torre, con annesse stanze e magazzini per la guarnigione, circondata da un semplice fossato. Questo embrione di castello venne demolito dai Gonzaga" (Cortesi, 2007, p. 246). Il primo fortilizio sopravvisse infatti fino al 1371, quando Feltrino Gonzaga (Mantova, ca. 1330 - Padova, 1374), investito del feudo nel 1354, diede inizio al ramo cadetto di Novellara e avviò la sua demolizione e l'ideazione dell' attuale rocca (Bertolani, 1965, pp. 49-54).
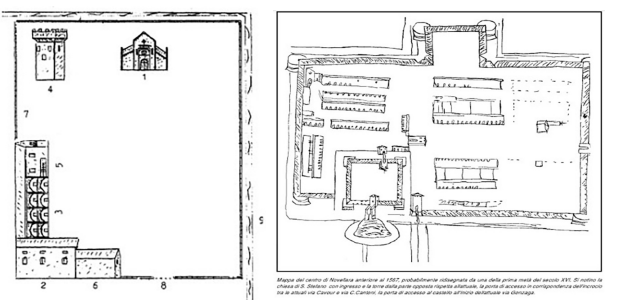

Figg. 1 e 2. Ricostruzione del castrum di Novellara nel XIII secolo (S. Coiroldi in Barilli, 1999) e, a destra, una raffigurazione della Rocca nell' assetto cinquecentesco (Davolio, 1825).

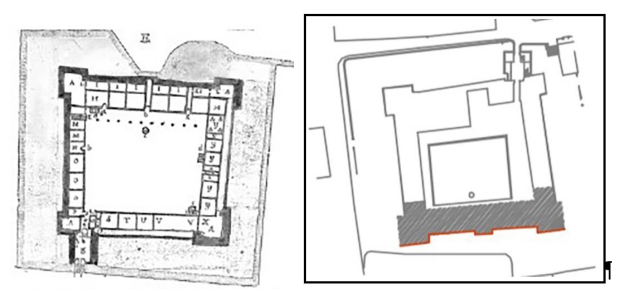

Figs. 3 e 4. Pianta settecentesca (P. Sigismondi) e pianta attuale con indicazione della facciata nord.

Soltanto la turris vetus fu risparmiata dall'abbattimento, non è noto se inglobata o meno nell'area della nuova costruzione ${ }^{2}$. La rocca, nella configurazione tuttora apprezzabile, dotata di quattro corpi e altrettante torri angolari (Fig. 2), è stata costruita da Guido Gonzaga, a partire dalla seconda metà del Trecento. Alla nobile fa- miglia -che si era rifugiata nel feudo di Novellara nel 1371- si devono le ulteriori modifiche, sia all'esterno che negli ambienti, documentate nel corso dei successivi XV e XVI secolo; fino alla costruzione, nel 1670, della torre cosiddetta "del Campanone", progettata da G.B. Sormani, che introduce alla corte interna e ha sostituito il rivellino medioevale. Di propietà comunale dal 1754 (Barilli, 1999, p. 24), la Rocca è oggi sede del municipio e ospita il Museo Gonzaga e il teatro, costruito nell'Ottocento, oltre ad una serie di servizi per la comunità, che la rendono un importante polo civile e culturale della vita contemporanea. Il complesso che, sin dal XVI secolo, aveva iniziato ad assumere le sembianze di un grande palazzo, è stato più volte restaurato e adattato ai nuovi usi (Figg. 3 e 4). Tuttavia la memoria del passato fortificato continua ad essere trasmessa, oltre che dal toponimo, anche dai resti della cinta muraria e delle torri angolari, nel tempo sottoposte a modifiche e a più recenti restauri di tipo conservativo, l'ultimo dei quali ha coinvolto la facciata occidentale (Guarisco, Locatelli, 1989, pp. 68-69).

\section{Fonti storiografiche e lettura diretta: un'osservazione di carattere metodologico}

Il contributo riporta gli esiti di una lettura stratigrafica effettuata sulla facciata nord ${ }^{3}$ - l'unica parte non ancora restaurata- che ha permesso di comprendere le diverse fasi costruttivotrasformative del fronte e di porle in una sequenza cronologica relativa. Lo scopo della stratigrafia è infatti quello di individuare le tracce e di comprendere l'origine delle modifiche architettoniche e funzionali rilevate (Doglioni, 1997). L'osservazione diretta delle tracce materiali ha rivelato il lento processo di trasformazione della Rocca da fortilizio a palazzo residenziale. Ma lo studio ha anche consentito una riflessione sul cambiamento di carattere dell'edificio, che nel tempo a assunto un'identità complessa, dovuta tanto ad interventi architettonici altamente qualificati quanto a piccole trasformazioni, che tuttavia suscitano interesse in termini di cultura materiale. Ad uno stato di conservazione estremamente differenziato nei diversi settori che compongono questo fronte, lungo quasi novanta metri, si aggiunge e un grado atrettanto variabile di 
permanenza di caratteristiche fortificate. Per ragioni di correttezza metodologica, le osservazioni stratigrafiche sono state condotte autonomamente e in una fase precedente l'analisi delle fonti indirette, affinchè fossero distinte le interpretazioni basate sulla sola osservazione diretta delle superfici architettoniche, rispetto ai risultati della ricerca documentale. Si è così cercato di garantire alla lettura diretta un approccio screvro da condizionamenti di tipo storiografico. La successiva acquisizione di informazioni, derivate dalla disanima della pubblicistica, ha fornito un quadro di riferimento ex post, certamente indispensabile ad associare la sequenza stratigrafica (o cronologia relativa) a elementi di datazione assoluta di origine documentale, secondo un'integrazione di metodiche analitiche qui sperimentata $e$ in seguito collaudata (Squassina, 2014, 2018). Cionondimeno, per agevolare la lettura del resoconto stratigrafico, in questa sede inevitabilmente sintetico, alcune note di inquadramento storico sono associate alle fasi individuate stratigraficamente e organizzate in forma di successione di fasi storiograficamente documentate, con dati e citazioni correlate a figure o ad eventi che hanno coinvolto l'architettura della Rocca.

\section{Riepilogo delle osservazioni stratigrafiche effettuate sul fronte nord della Rocca}

Il processo di stratificazione della facciata nord della Rocca viene qui sinteticamente illustrato, distinguendo le osservazioni nei diversi settori (torre nord-est, settore sinistro-est, torre centrale, settore destro-ovest, torre nord-ovest) e organizzandole secondo la sequenza stratigrafica relativa individuata. Propedeutica alla lettura stratigrafica vera e propria, è stata l'analisi dei diversi tipi di murature individuati, secondo un approccio metodologico ulteriormente sviluppato da chi scrive negli anni recenti (Squassina, 2011), che ha consentito di correlare le diverse murature alle relative fasi stratigrafiche ${ }^{4}$. Il risultato delle osservazioni è una sequenza stratigrafica complessiva, che costituisce una prima cronologia relativa delle fasi trasformative individuate (Fig. 9), la quale può essere ulteriormente specificata, in termini di cronologia assoluta, grazie al riferimento - qui contestualmente riportato- alle fonti storiche.

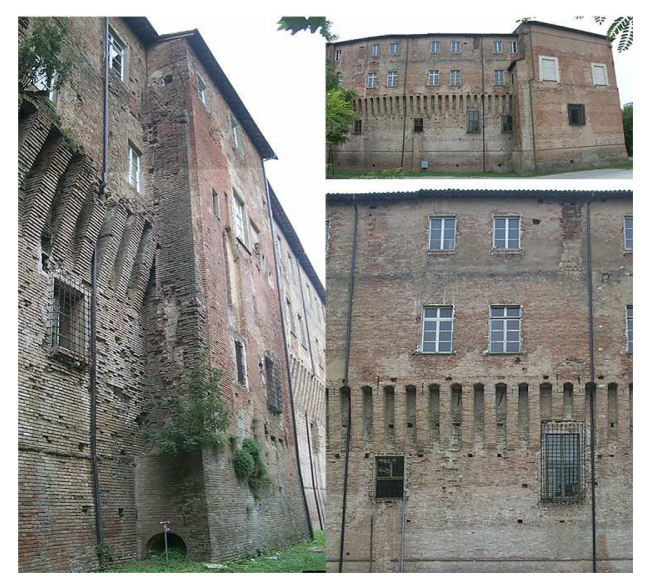

Fig. 5. Viste del fronte nord della rocca da cui se ne evince la natura stratificata: a sinistra la torre centrale con le tracce del ponte levatoio; a destra, vista e dettaglio del settore-torre n-o, in cui sono riconoscibili i resti dei merli ghibellini iniziali, al primo livello.

\subsection{Descrizione sintetica delle fasi stratigrafi- camente individuate (con note di inquadra- mento storico)}

Elemento caratterizzante la facciata nel suo complesso è la diversa condizione delle parti: le due torri laterali sono state restaurate in epoca relativamente recente, con rifugatura dei giunti di malta che ha reso poco leggibile la superficie. La torre centrale, palinsesto di elementi tipici del fortilizio e modeste stratificazioni d'uso, separa i due settori est ed ovest; il primo, alterato da degrado e demolizioni, ha carattere ruderizzato ma presenta le tracce delle modifiche architettoniche più rilevanti. Mentre il settore ovest rivela una stratificazione minuta e ordinata e conserva gran parte della cortina iniziale, un tempo merlata, con beccatelli ancora presenti.

\section{Fase 0. La rocca inziale (secoli XIV-XV)*}

Nel complesso è possible individuare la parte del fortilizio iniziale, la cui muratura si estende per un solo livello ed è composta da mattoni di colore rosso-bruno (dimensioni medie 6,3 x 13,5 x $30,2 \mathrm{~cm}$ ) e giunti di malta di calce piuttosto alti (14-23 mm), accuratamente spianati, di colore 
avorio chiaro, con sabbia fine, calcinaroli ed inerti più grandi, di colore chiaro, leggermente rosato.

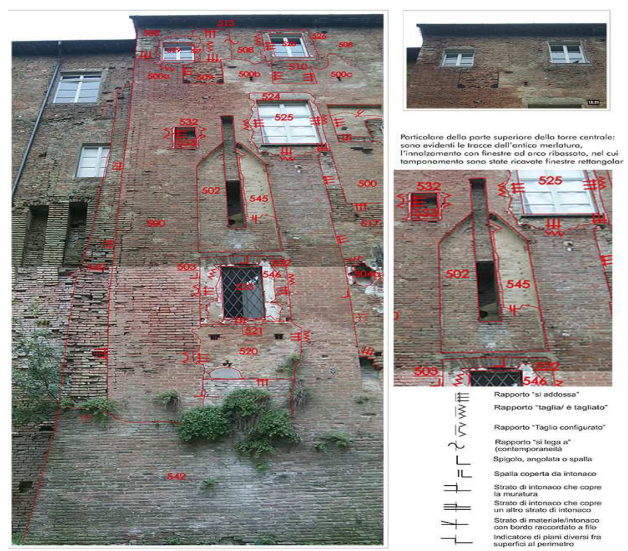

Fig. 6. Stratigrafia della torre centrale: dettaglio delle tracce della sede del bolzone del ponte levatoio e delle apeture ad arco bardellonato, successivamente riconfigurate, così come accaduto a quelle presenti nelle altre torri (C-S: elaborato da Crescini-Squassina).

Questo impianto -costituito da quattro corpi di fabbrica disposti intorno ad un cortile rettangolare con quattro torri rettangolari aggettanti agli angoli, rivellino a protezione dell'ingresso e mura esterne a scarpa- è tipico del castello medioevale di pianura (Perogalli, 1972, p. 21) e si sviluppa qui su di una superficie di circa 7750 $\mathrm{mq}^{5}$, caratterizzato da due ponti levatoi sui fronti sud e nord, di cui si scorgono ancora le tracce. Sul fronte nord si mantiene il basamento a scarpa, delimitato da un redondone sagomato a toro, beccatelli con merli ghibellini, ancora in parte distinguibili, seppure inglobati nell'innalzamento successivo (Fig. 5) e piccole aperture ad arco bardellonato. Nella torre centrale, la cui muratura risulta ammorsata a quella dei settori est ed ovest (nel primo livello), compaiono le sedi dei bolzoni sia del ponte levatoio che della pusterla pedonale (Fig. 6). Le apeture ad arco bardellonato del primo fortilizio hanno subito tamponamenti e/o trasformazioni, legate alle differenti funzioni che il castello ha assunto nel tempo. In particolare, l'ambito della torre centrale ha registrato una sorta di "scadimento" simbolico, da secondo ingresso fortificato ad ambiente di residenza, infine a zona di servizi, con annes- so un necessario aggettante. Mentre nei settori est ed ovest (Figg. 7 e 8) sopravvivono i beccatelli iniziali, che si distinguono dai successivi per la presenza di caditoie, tipiche della difesa piombante di origine medioevale (Perogalli, 1972, p. 24; Palloni, 1992, pp. 22-34).

* Note di inquadramento storico - la rocca dei Gonzaga (XIV-XV secolo): l'ambizioso progetto di Feltrino Gonzaga per un nuovo 'castrum cum subterraliis et aliis aedificis' (Barilli, 1999, p. 23) fu attuato dal figlio Guido (1340-1399) il quale, fra il $1385-86$ e il 1452 , procedette allo scavo dei fossati, alla formazione delle fondazioni e al consolidamento dei terrapieni attorno al castello (Bertolani, pp. 49-54). Si deve invece ai suoi successori il completamento; secondo una versione storiografica al nipote Giacomo, nel 1450 (1399-1441, Barilli, 1999, p. 12), ovvero ai fratelli Giorgio e Franco (ndr Francesco I, 1420 ca.-1484), nel 1464, "così da antichissimo libro in Archivio' (Davolio, 1825, p. 31). La rocca, nella configurazione quattrocentesca con 'muraglie a scarpa ... di 30 testate [...] era sostanzialmente una robusta fortezza [...] però sia Giacomo che Francesco I [...] avevano fatto costruire appartamenti all'interno [...] con un gusto prettamente rinascimentale" (Barilli, 1999, p. 23).

\section{Fase 1. Riparazioni remote (secolo XV?)*}

Il basamento mostra in più parti discontinuità costruttive, ampliamenti e integrazioni murarie, segno di riparazioni nel tempo, peraltro documentate sin dal XV secolo.

* Note di inquadramento storico - assedi, riparazioni e rinforzi: la rocca subì un lungo assedio nel 1452, durante la guerra fra i Visconti e i Veneziani, terminata con la pace di Lodi nel 1454. Rientrati inpossesso di Novellara, i Gonzaga procedettero alla riparazione dei danni: "Si risarcirono i fabbricati, si scavarono di nuovo le fosse, e si rifecero i terrapieni, che circondavano il vecchio Castello" (Davolio, 1825, pp. 40-42).

Altri rinforzi alla rocca sono documentati alla fine del Quattrocento (1494-99) e attribuiti a Giampietro (1469-1515) il quale, minacciato dalle lotte di confine, 'riattò le fortificazioni che 
circondavano il Castello, ne riempì le fosse d'acqua' (Davolio, 1825, p. 56).

\section{Fase 2. Primo innalzamento: la loggia (secolo XVI)*}

Nel campo sinistro si osserva l'interfaccia di demolizione di gran parte dei merli e, sopra i beccatelli, si innesta un primo innalzamento, contenente una teoria di 7 archi a tutto sesto su pilastri (successivamente tamponati ed in parte trasformati in finestre rettangolari, Fig. 7) e tre archi più slanciati verso la torre centrale, con tracce di rottura superficiale sugli archivolti, presumibilmente appartenuti ad una parte aggettante, in seguito demolita. Questo innalzamento si distingue anche per una diversa muratura, in mattoni di colore rosso-aranciato $(5,8 \times 13 \mathrm{x}$ $27,8 \mathrm{~cm}$ ), con giunto arretrato di malta di calce di colore giallo, con sabbia fine gialla e rari inerti più grandi di colore grigio-giallastro. A questa fase sono correlati anche venti dei ventisei beccatelli presenti al primo livello del settore ovest (Fig. 8), caratterizzati dall'assenza di caditoie (che compaiono invece in sei beccatelli precedenti e in quelli presenti nel settore est) e sembrano quindi frutto di una ricostruzione dovuta più a ragioni architettoniche che difensive, confermando cioè l'appartenenza alla roccaresidenza.

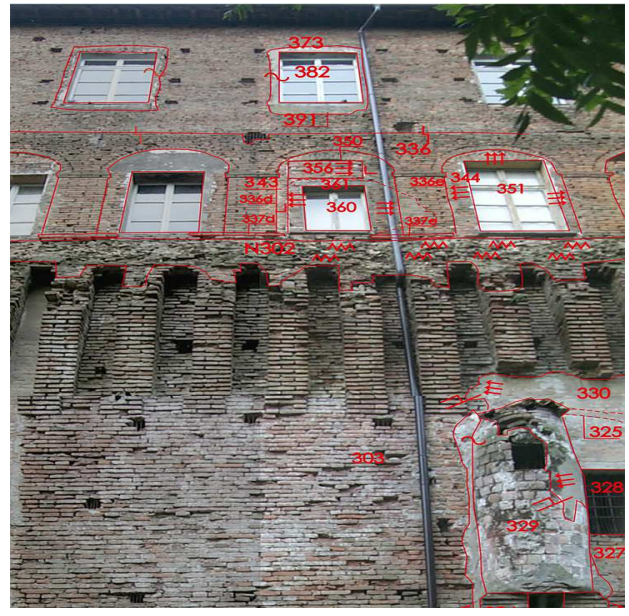

Fig. 7. Gli archi tamponati della loggia, costruita sui resti della merlatura iniziale, nel settore est (C-S).
* Note di inquadramento storico - da fortezza a dimora signorile (XVI secolo): questa fase si identifica con l'innalzamento storicamento documentato agli inizi del XVI secolo. Se in una stima di divisione fra Guido e Giampietro Gonzaga dell'anno 1500 , si descrive la rocca di Novellara ancora solo "alta 23 braccia a contare dal volto sotterraneo, e non aveva che gli appartamenti a pianterreno" (Davolio, 1825, p. 61. Ndr: il braccio reggiano corrisponde a circa $63,3 \mathrm{~cm}$ ); con il privilegio imperiale del 1501, Novellara assunse il titolo di contea e il primo conte, Gian Pietro Gonzaga, dispose un restauro della Rocca allora estesa su un solo piano. L'innalzamento fu compiuto da Costanza da Correggio, vedova di Alessandro Gonzaga. Nel suo elenco di opere da realizzarsi, inviato per approvazione al Conte Giulio il 22 Novembre 1541, figura "un secondo piano con loggia e sala [...] sul vecchio fabbricato della Rocca (scrittura di contratto con l'architetto Mastro Annibale Guberti 14 Marzo 1552)" (Davolio, 1825, pp. 149-50). Con quest'opera iniziò il processo di trasformazione della Rocca da fortilizio a dimora, sede ufficiale della corte signorile; un processo che era iniziato nel 1518, quando "messer Antonio e messer Latino con due giovini tutti di Correggio dipingevano [...] la camera del torrione vecchio appena ristrutturati" (Barilli, 1999, p. 23). La trasformazione proseguì con Alfonso I e Camillo Gonzaga, e poi con Francesco II, committenti dell'artista di corte Lelio Orsi per i dipinti e la supervisione delle opere compiute, fra il 1561 e il 1567 , a decorazione degli ambienti interni e della loggia, oltre che del teatro, costruito nel 1568.

\section{Fase 3. Innalzamento del secondo piano con finestre (secolo XVII)*}

Ad una fase successiva appartiene un secondo innalzamento, rappresentato da una muratura complanare (nel settore ovest) o leggermente arretrata (mediante risega nel settore est) rispetto alla sottostante. Questa muratura -caratterizzata da mattoni rossi $(5,9 \times 13,5 \times 29,7 \mathrm{~cm})$, con giunto di malta di colore beige chiaro, leggermente grigio, con sabbia finissima, piccoli inerti dello stesso colore, e rari inerti più grandi e chiari (spessore $20 \mathrm{~mm} \mathrm{ca}$.) - contiene una teoria di finestre rettangolari con spalle in muratura e 
piattabanda in mattoni disposti di fascia (aperture in seguito riconfigurate, Fig. 8). Attualmente il settore destro risulta essere l'unica parte rivestita da tratti di intonaco (Fig. 4). Ma, in una nicchia della torre centrale, sono stati rilevati tre strati di intonaco, prova stratigrafica di tre fasi, in cui almeno alcune parti della rocca erano ricoperte da rivestimento.

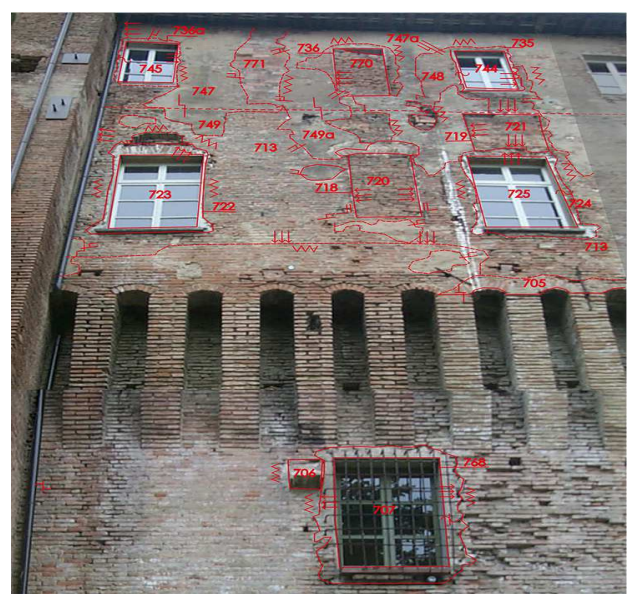

Fig. 8. Settore ovest: in alto, il secondo piano e alcune delle finestre - in seguito riconfigurate - correlati alla terza fase. In basso si osservano anche i beccatelli iniziali con caditoia (i primi sei da sinistra) e due di quelli parzialmente ricostruiti nel corso del primo innalzamento e privi di caditoie (C-S).

* Note di inquadramento storico - le modifiche seicentesche: "Sarebbe passato un secolo prima che si eseguissero altri lavori consistenti. Nel 1670 Alfonso II fece innalzare da G.B.Sormani la torre detta "Campanone" sulla porta esterna della rocca, "[...] ornata di guglie, di lanterna e di cupola a squama di pesce con due giri di ben lavorate ringhiere [...]", ed effettuare una nuova sistemazione degli appartamenti in particolare del secondo piano del lato nord" (Barilli, 1999, p. 23). Ad Alfonso II (1616-1678) si deve anche l'attribuzione al mastio della funzione di prigione, con accesso dal lato ovest della rocca.

\section{Fasi 4. Riconfigurazioni successive (post 1754)*}

A fasi successive, non facilmente correlabili le une alle altre appartengono interventi di modifica nelle aperture, aggiunte di carattere funziona- le (corpi aggettanti, un necessario e una sorta di garitta), fino alle più recenti sottomurazioni e rifugature caratterizzate da malta cementizia. Si tratta di modifiche per lo più minute, non sempre architettonicamente configurate, che testimoniano l'adattamento d'uso resosi necessario nel tempo, che ha declassato il fronte nord da secondo accesso principale a fronte posteriore. A queste fasi è correlabile anche l'ultimo strato di intonaco rilevato su settore e torre ovest, in seguito alla riconfigurazione delle coperture.

*Note di inquadramento storico - dopo i Gonzaga, La Contea di Novellara passò agli Este nel 1737, dopo la morte dell'ultimo conte Filippo Alfonso Gonzaga (1728); per la rocca iniziò un periodo di decadenza: "furono tolti i ponti levatoi, chiusa la sortita a settentrione, [...] si disfò una parte della quadra che guarda a mezzodì, poi i corridoi a mattina ed a meriggio coll'abbassare i tetti, si atterrarono i 4 torrioni agli angoli [...] si devastarono gli ultimi appartamenti del piano superiore [...] se ne affittarono le camere a poveraglia che ne imbrattò i muri, distrusse e portò via tutto ciò che potè" (Barilli, 1999, p. 23-24). La Rocca divenne proprietà comunale nel 1754 e fu adibita a destinazioni pubbliche, uffici, scuole e abitazione di dipendenti comunali. Queste ultime modifiche comportarono l'obliterazione di gran parte dei caratteri castellani, come i ponti levatoi e l'ingresso sul fronte settentrionale. Un ultimo intervento rilevante fu il teatro, costruito tra il 1862 ed il 1868 sul sedime del teatro cinquecentesco decorato da Lelio Orsi e demolito in quell'occasione, insieme ad altri ambienti gonzagneschi, come le cucine, la lavanderia e la legnaia (Barilli, 1999, p. 24).

\section{Conclusioni}

Le diverse fasi individuate hanno permesso di seguire il processo evolutivo della facciata (e, con questa, dell'edificio), che da manufatto chiuso e fortificato, con aperture limitate ed a prevalente carattere difensivo (fase zero: la rocca), si è gradualmente "aperta" all'esterno, con la formazione di grandi finestre nella parte basamentale e logge ad arco ai livelli superiori. Il periodo in cui la rocca è divenuta prevalentemente residenza è caratterizzato da diverse fasi di riconfigurazione, 
alcune altamente connotate (la loggia), altre riguardanti elementi più minuti ma altrettanto interessanti, soprattutto le aperture, più volte ridimensionate, ed alcune aggiunte o modifiche funzionali (canne fumarie, necessari e vani di servizio con relative aperture), legate ad un diverso utilizzo degli ambienti interni e alla modifica dei modi d'uso e dell'abitare nel tempo.

Il passaggio da fortezza a residenza è enfatizzato dalla trasformazione dei sistemi di accesso, in particolar modo dal tamponamento delle porte carraia e pedonale appartenenti alla torre centrale, che avevano un ruolo fondamentale nella configurazione e nell'assetto distributivo della rocca. Il processo evolutivo dell'insieme è stato caratterizzato tanto da riconfigurazioni intenzionali e modifiche funzionali, quanto da eventi traumatici, testimoniati dalla demolizione di parte della struttura muraria merlata e dei beccatelli, non semplicemente inglobati nelle murature successive, ma in larga parte crollati o demoliti. Nel settore sinistro, soprattutto, si registrano ampie zone di crollo dei beccatelli e una condizione ruderizzata, oltre al fatto che la stratificazione risulta aver modificato in modo radicale l'assetto della facciata. Il settore destro (ovest) ha un carattere notevolmente diverso da quello del campo sinistro, con un maggior grado di configurazione e di decoro, che non diminuisce l'interesse stratigrafico. Questa commistione di alterazioni naturali e antropiche rende la facciata nord un palinsesto di particolare interesse e induce considerazioni sulle modifiche del carattere dell'edificio, che nel tempo ha assunto un'identità complessa, legata al ruolo delle diverse parti, alla permanenza o meno del carattere fortificato - non sempre evidente e decifrabile alla valenza architettonica e formale di alcune aggiunte di pregio ma anche al significato stratigrafico di elementi minori, non sempre accuratamente configurati. Le tracce tuttora leggibili costituiscono una risorsa in termini conoscitivi e di memoria ma anche una condizione progettualmente impegnativa. La permanenza dei segni in assenza di alterazioni è l'obiettivo principe di un approccio conservativo, che non può che misurarsi con l'eterogenea caratterizzazione delle singole parti. Ne scaturisce una riflessione sulla possible strategia progettuale e sull'approccio operativo conseguente, che non può eludere questa molteplicità di aspetti, che costituisce il fondamentale carattere acquisito della facciata, suggerendo una forma di progettualità articolata su diversi registri, capace di dare una risposta conservativa a contesti architettonici e materiali così differenziati per aspetto e grado di conservazione.

\section{Note}

1 "In funzione antisaracenica erano state costituite da Berengario II, nel 952, le marche Arduinica, Aleramica ed Obertenga, in un territorio che si estendeva ad arco dalle Alpi Marittime fino al Tirreno. [...] Dal ceppo obertengo dei marchesi di Toscana ebbe origine la numerosa e longeva discendenza dei marchesi Malaspina, il cui dominio feudale era stato ufficialmente sancito dal diploma di investitura di Federico Barbarossa [...] nel 1164. [...] Ma questo nome, come altri attribuiti a signori di quel tempo quali [...] Malnipote e Malapresa, è da riferirsi più verosimilmente al modo non sempre onesto di amministrare i loro feudi [...]" (Capecchi, 1990).

2 "A proposito di torri, non si è ancora potuto stabilire se la torre dei Malapresa fosse all'interno del Catelloncolo o nell'area dell'attuale cortile della rocca." (Barilli, 1999, p. 11).

${ }^{3}$ Lo studio stratigrafico è stato condotto da chi scrive per incarico del Comune di Novellara, in collaborazione con l'architetto Alba Crescini e nell'ambito di un progetto preliminare di consolidmento e restauro (ing. Stefano Valenti).

${ }^{4}$ Per ogni settore è stata anche elaborata una scheda di archiviazione veloce delle singole USM rilevate (Brogiolo, 1988); per ragioni di sintesi le schede non sono riportate in questa sede.

${ }^{5} \mathrm{Cfr}$. www.comune.novellara.re.it. 


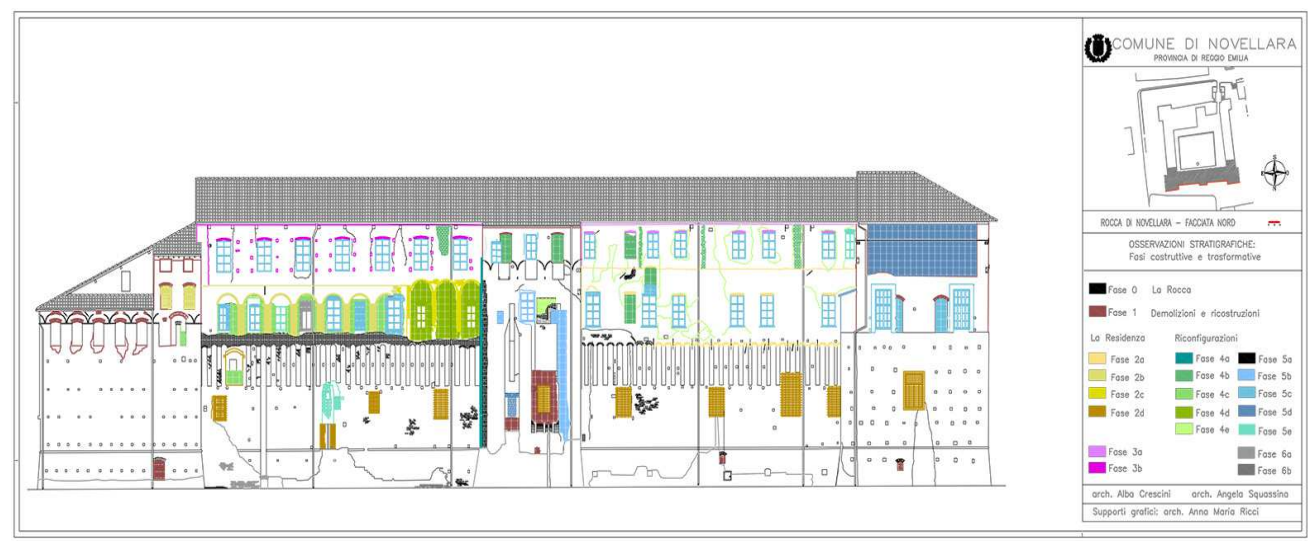

Fig. 9. Schema riepilogativo delle diverse fasi costruttive individuate (A. Squassina, A.Crescini).

\section{Bibliography}

Barilli, G.P. (1999). Vie strade Piazze di Novellara, RP-GPB, Novellara.

Bertolani Del Rio, M. (1959). I Castelli reggiani, Comune Reggio Emilia, Milano.

Brogiolo, G.P. (1988). Archeologia dell'edilizia storica, New Press, Como.

Cortesi, P. (2007). I Castelli dell'Emilia Romagna, Newton Compton, Roma.

Davolio, V. (1825). Memorie Storiche della Contea di Novellara e dei Gonzaghi che vi dominarono.

Doglioni, F. (1987). Stratigrafia e restauro, Lint, Trieste.

Fiorini, A. (2012). "I castelli della Romagna: materiali costruttivi, elementi architettonici e progettazione", in Redi, F.; Forgione, A., coord., VI Congresso Nazionale di Archeologia Medievale. L'Aquila, All'Insegna del Giglio, Firenze, pp. 93-99.

Grisoni, M.M.; Scharf, G.; Squassina, A.P. (2014). "La divisione tra Francesco e Guido Visconti (1473). Dalla fonte d'archivio al rilievo dell' architettura del castello di Somma Lombardo", in Grisoni, M. coord., Conoscere per tutelare e vlorizzare il paesaggio storico, Debatte Ed., Livorno, pp. 56-67.

Guarisco, G.; Locatelli, V. coord. (1989). "Novellara, la rocca Gonzaga (il progetto di conservazione, 1988)", in $M$. Dezzi Bardeschi. Conservazione e Metamorfosi. Cosmogonie, bestiari, architetture, 1978-1988. Catalogo della mostra, Macerata, 12-24 maggio 1989, Alinea, Firenze, pp. 68-69.

Palloni, D. (1992). "La difesa piombante e le artiglierie nevrobalistiche", in La sicurezza dell'esistere. Le architetture fortificate al tempo di Lorenzo, Istituto Italiano dei Castelli, Firenze, pp. 22-34.

Panero, F.; Pinto G., coord. (2009). Castelli e fortezze nelle città e nei centri minori italiani (secoli XIII-XV). Atti del Convegno.Cherasco 15-16/11/2008, CIM, Cherasco.

Perogalli, C. (1972). Castelli e rocche di Emilia e Romagna, Goerlich, Milano.

Rotelli, G. (1909). Principali Castelli della Provincia di Reggio Emilia, Reggio Emilia.

Squassina, A. (2011). "Le variazioni dimensionali e delle lavorazioni di superficie dei laterizi a Venezia: la chiesa dei Carmini come sequenza cronologica", in Doglioni, F.; Mirabella Roberti, G., coord, Venezia. Forme della costruzione forme del dissesto, Cluva, Venezia, pp. 67-88.

Squassina, A. (2018). "Fortificazioni veneziane. Lo studio delle trasformazioni per il restauro della torre di Mestre come approccio conservativo", in Marotta, A.; Spallone, R., coord., FORTMED 2018. Proceedings of the International Conference on Modern Age Fortification of the Mediterranean Coast, Politecnico di Torino, Torino, vol. VIII, pp. 409-416. 